\title{
Status of indirect searches in the PAMELA and Fermi era
}

\author{
Aldo Morselli* \\ INFN Roma Tor Vergata, Italy \\ E-mail: aldo.morselli@roma2.infn.it

\section{Igor V. Moskalenko} \\ Hansen Experimental Physics Laboratory and Kavli Institute for Particle Astrophysics and \\ Cosmology, Stanford University, USA \\ E-mail: imosestanford.edu
}

The detection of $\gamma$-rays, antiprotons and positrons due to pair annihilation of dark matter particles in the Milky Way halo is a viable indirect technique to search for signatures of supersymmetric dark matter where the major challenge is the discrimination of the signal from the background generated by standard production mechanisms. The new PAMELA antiproton data are consistent with the standard secondary production and this allows us to constrain exotic contribution to the spectrum due to neutralino annihilations. In particular, we show that in the framework of minimal supergravity (mSUGRA), in a clumpy halo scenario (with clumpiness factor $\geq 10$ ) and for large values of $\tan (\beta) \geq 55$, almost all the parameter space allowed by WMAP is excluded.

Instead, the PAMELA positron fraction data exhibit an excess that cannot be explained by secondary production. PPB-BETS and ATIC reported a feature in electron spectrum at a few hundred $\mathrm{GeV}$. The excesses seem to be consistent and imply a source, conventional or exotic, of additional leptonic component.

Here we discuss the status of indirect dark matter searches and a perspective for PAMELA and Fermi $\gamma$-ray space telescope (Fermi) experiments.

Identification of dark matter 2008

August 18-22, 2008

Stockholm, Sweden

\footnotetext{
*Speaker.
} 


\section{Antiproton to proton ratio data}

The PAMELA (a Payload for Antimatter Matter Exploration and Light-nuclei Astrophysics) experiment is a satellite-borne apparatus designed to study charged particles in the cosmic radiation with a particular focus on antiparticles (antiprotons and positrons) [1]. The PAMELA antiproton data [2] are shown in figure 1 together with the antiproton flux expected from standard secondary production. Cosmic ray propagation and production of secondary particles and isotopes is calculated using the GALPROP code [3]. The lines show the minimal and maximal fluxes as calculated in models with different propagation parameters tuned to match the boron-to-carbon ratio in cosmic rays $([4,5,6,7])$. The antiproton data collected by PAMELA [2] and BESS [8] are consistent with each other and with predictions for secondary antiproton flux thus excluding a strong antiproton signal from exotic processes. Figure 2 is made in the framework of minimal supergravity (mSUGRA) by fixing the less sensitive parameters $A_{0}, \tan \beta$ and $\operatorname{sign}(\mu)=+1$ and in the case of a clumpiness factor 10 and $\tan (\beta)=55$. Following the analysis in [6], the region below the line in figure 2 can be excluded based on antiproton data. For larger value of $\tan (\beta)$ the excluded parameter space is even larger, while for lower values the capability of the antiprotons flux to probe the mSUGRA scenario is very weak $([6,9])$.

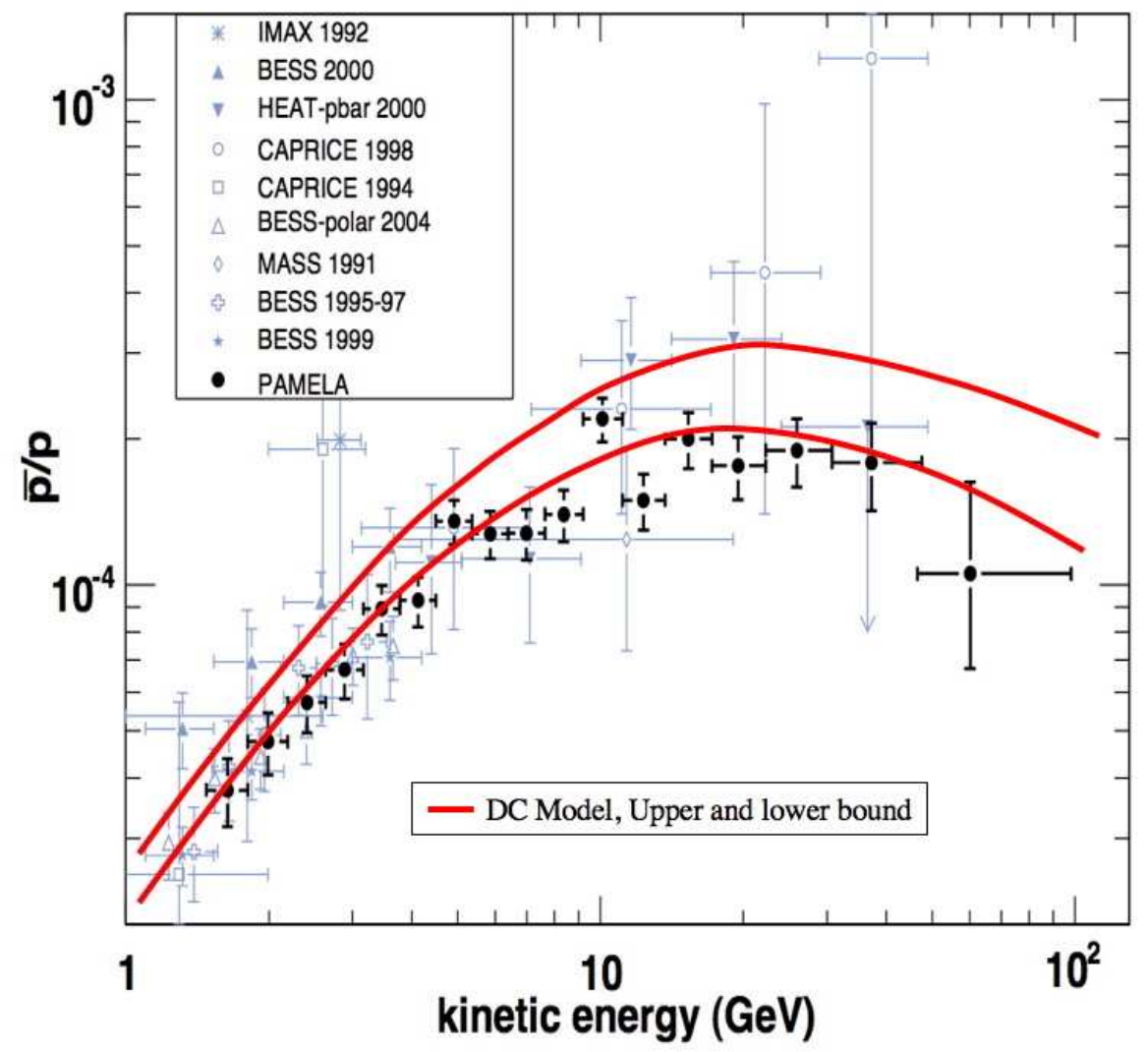

Figure 1: The antiproton-to-proton flux ratio as measured by PAMELA [2]. The lines show an approximate range expected for the standard secondary production $[4,5,6,7]$. 


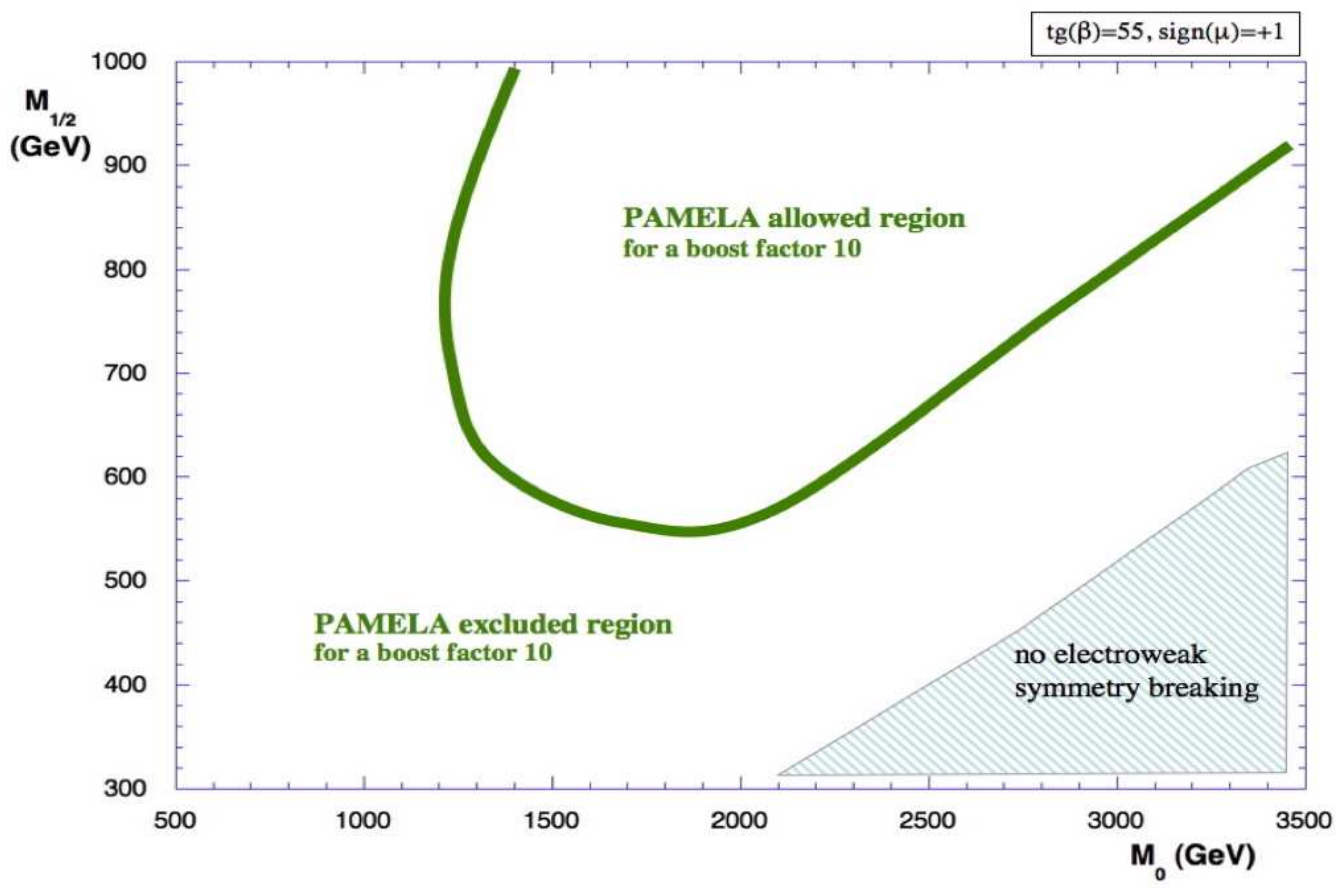

Figure 2: PAMELA excluded region in a clumpy halo scenario for a boost factor 10 in the framework of minimal supergravity (mSUGRA) in the case of $\tan (\beta)=55$.

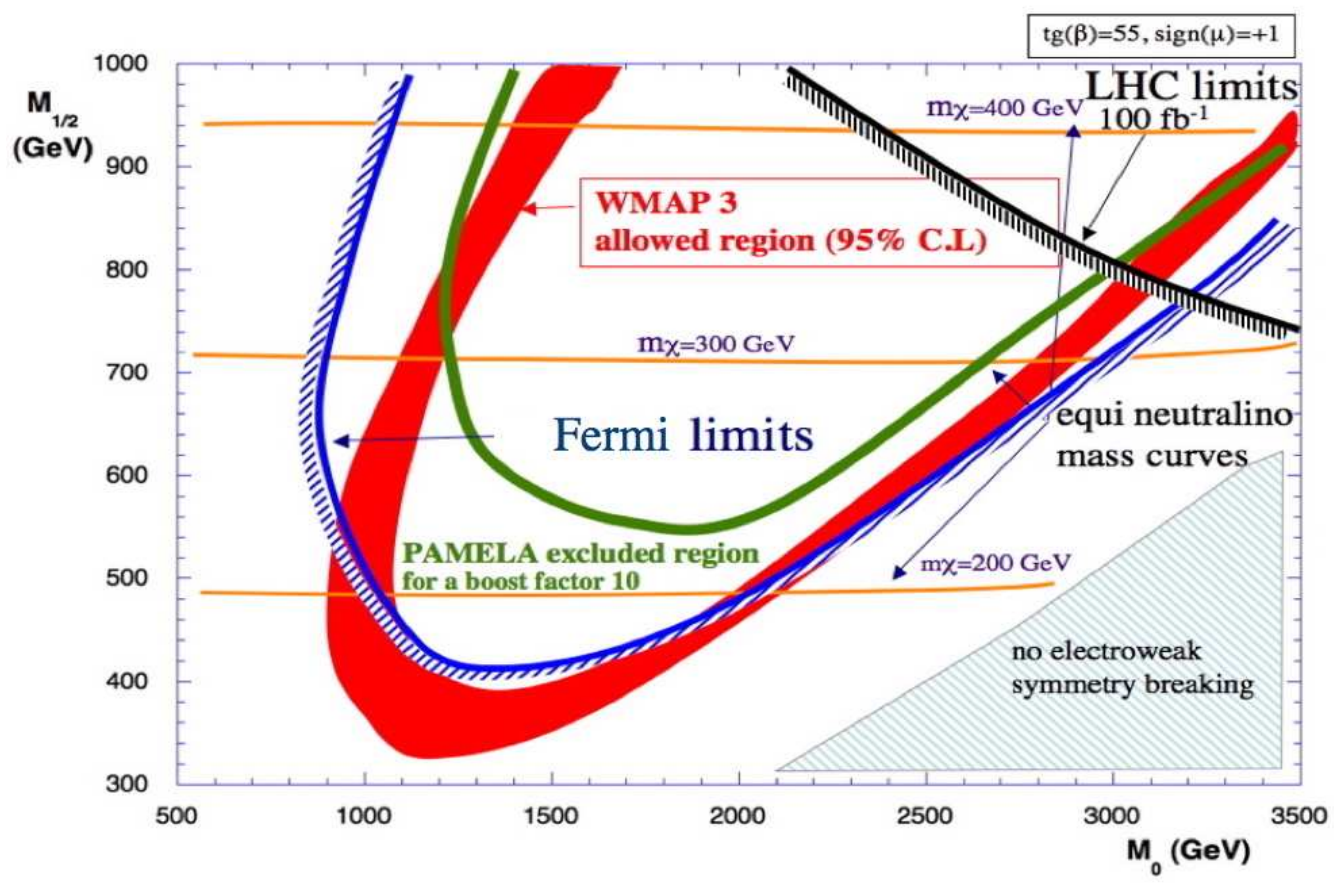

Figure 3: Sensitivity plot for observation of mSUGRA for LHC and Fermi 
This result can be compared with estimates based on Fermi five-years sensitivity to WIMP annihilation photons (continuum spectrum) from the Galactic center as shown in figure $3[10,11]$. The red band is the cosmologically allowed region by WMAP [12]; the region above the blue line $\left(M_{\text {WIMP }} \sim 200 \mathrm{GeV}\right)$ is not observable by Fermi due to the higher WIMP mass as one moves to higher $M_{1 / 2}$. The dark matter halo used for the Fermi indirect search sensitivity estimate is a truncated Navarro, Frank and White (NFW) halo profile. For steeper halo profiles (like the Moore profile) the Fermi limits move up, covering a wider WMAP allowed region, while for less steep profile (like the isothermal profile) the Fermi limits move down, covering less WMAP allowed region. The LHC accelerator limits are from [13].

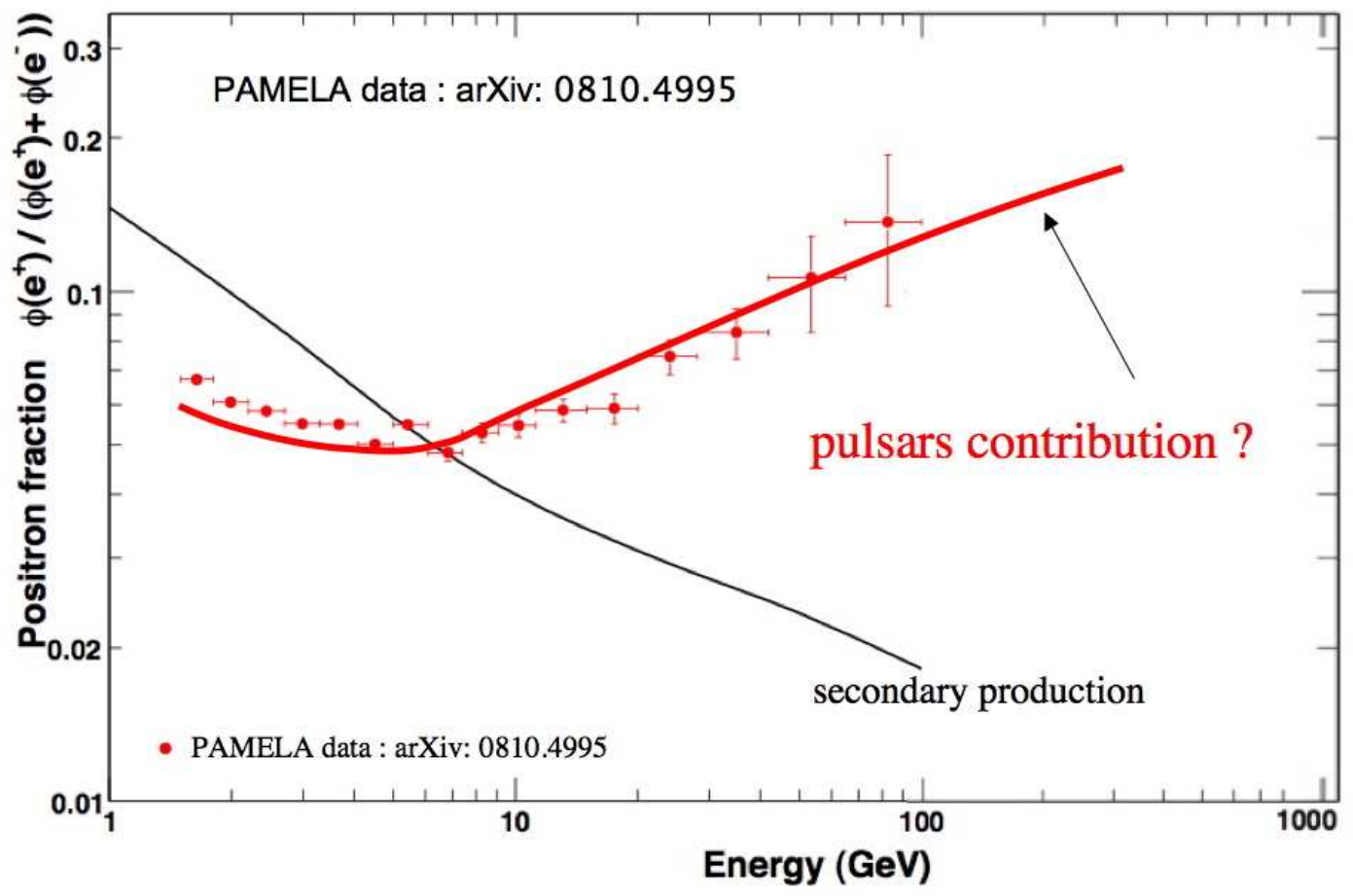

Figure 4: PAMELA data and a possible contribution from pulsars (scaled from [16]). Black solid line shows the background from secondary positrons in cosmic rays from GALPROP $[4,6]$.

\section{Positron fraction and electron excess}

Contrary to the antiproton to proton ratio data, the PAMELA positron fraction data [14] exhibit an excess above $\sim 10 \mathrm{GeV}$ that cannot be explained by secondary production $[4,6]$. We note that the change in the positron fraction data below $\sim 10 \mathrm{GeV}$ is probably due to the solar modulation (e.g., [15]) and change in the polarity of the solar magnetic field compared to the previous cycle. The temptation to claim the discovery of dark matter is strong, but there are competing astrophysical sources, such as pulsars, that can give strong flux of primary positrons and electrons $[16,17,18]$. In figure 4 the PAMELA data are shown with a possible pulsar contribution scaled from [16].

An independent confirmation that something interesting is going on with leptons in cosmic rays came from measurements of high-energy electrons. The cosmic-ray electron flux has not been 
measured very well in the past and especially at very-high energies because of the very steep spectrum and thus the need for high rejection power and long exposure. Simulations of the electron propagation from local sources [26] has shown that features in the electron spectrum may be expected in the $\mathrm{TeV}$ range where the flux of Galactic cosmic-ray electrons gradually steepens. On the other hand, annihilation of Kaluza-Klein particles may produce spectral features in sub-TeV range [20]. The first indication of a feature (or excess) in the electron spectrum at a few hundred $\mathrm{GeV}$ came from PPB-BETS flight a couple of years ago [23]. A recent confirmation of the excess by ATIC [24] gives more confidence that this is not an instrumental artefact.

How can one distinguish between the contributions of pulsars and dark matter annihilations? Most likely, a confirmation of the dark matter signal will require a consistency between different experiments and new measurements of the reported excesses with large statistics. The observed excess in the positron fraction should be consistent with corresponding signals in absolute positron and electron fluxes in the PAMELA data and all lepton data collected by Fermi [19]. Fermi has a large effective area and long projected lifetime, 5 years nominal with a goal 10 years mission, which makes it an excellent detector of cosmic-ray electrons up to $\sim 1 \mathrm{TeV}$ [25]. Fermi measurements of the total lepton flux with large statistics will be able to distinguish a gradual change in slope with a sharp cutoff with high confidence [20]. The latter, as shown in figure 5, can be an indication in favor of the dark matter hypothesis. A strong leptonic signal should be accompanied by a boost in the $\gamma$-ray yield providing a distinct spectral signature detectable by Fermi [21, 22]. Antiproton data with higher statistics and at higher energies collected by PAMELA could also give us some clue.

If the sources of the excess positrons are pulsars, they should be quite close to us and, therefore, may be detectable in $\gamma$-rays with Fermi. In this case, one has to expect broader features in the electron and positron spectra without sharp cutoffs. Meanwhile, the proposed test of the anisotropy in the total lepton (electron+positron) flux [18] may not work. First, the predicted anisotropy is very small, at the fraction of a per cent. Second, the so-called heliospheric modulation strongly affects the flux of cosmic-ray species below $20-50 \mathrm{GeV}$. The extended heliospheric magnetic field and the solar wind may affect the arriving directions of cosmic-ray particles at even higher energies. Therefore, even if the anisotropy is observed it may be connected with configuration of the heliospheric magnetic field rather then due to the local sources of primary leptons.

\section{Conclusion}

Recent accurate measurements of cosmic-ray positrons and electrons by PAMELA, PPBBETS, and ATIC have open a new era in particle astrophysics. The observed features or excesses break a boring single-power-law behavior of the cosmic-ray spectrum. Their exotic origin has to be confirmed by complimentary findings in $\gamma$-rays by Fermi and atmospheric Cherenkov telescopes, and by LHC in the debris of high-energy proton destructions. A positive answer will be a major breakthrough and will change our understanding of the universe forever. On the other hand, if it happens to be a conventional astrophysical source of cosmic rays, it will mean a direct detection of particles accelerated at an astronomical source, again a major breakthrough. In this case we will learn a whole lot about our local Galactic environment. However, independently on the origin of these excesses, exotic or conventional, we can expect very exciting several years ahead of us. 
I. V. M. acknowledges support from NASA Astronomy and Physics Research and Analysis Program (APRA) grant.

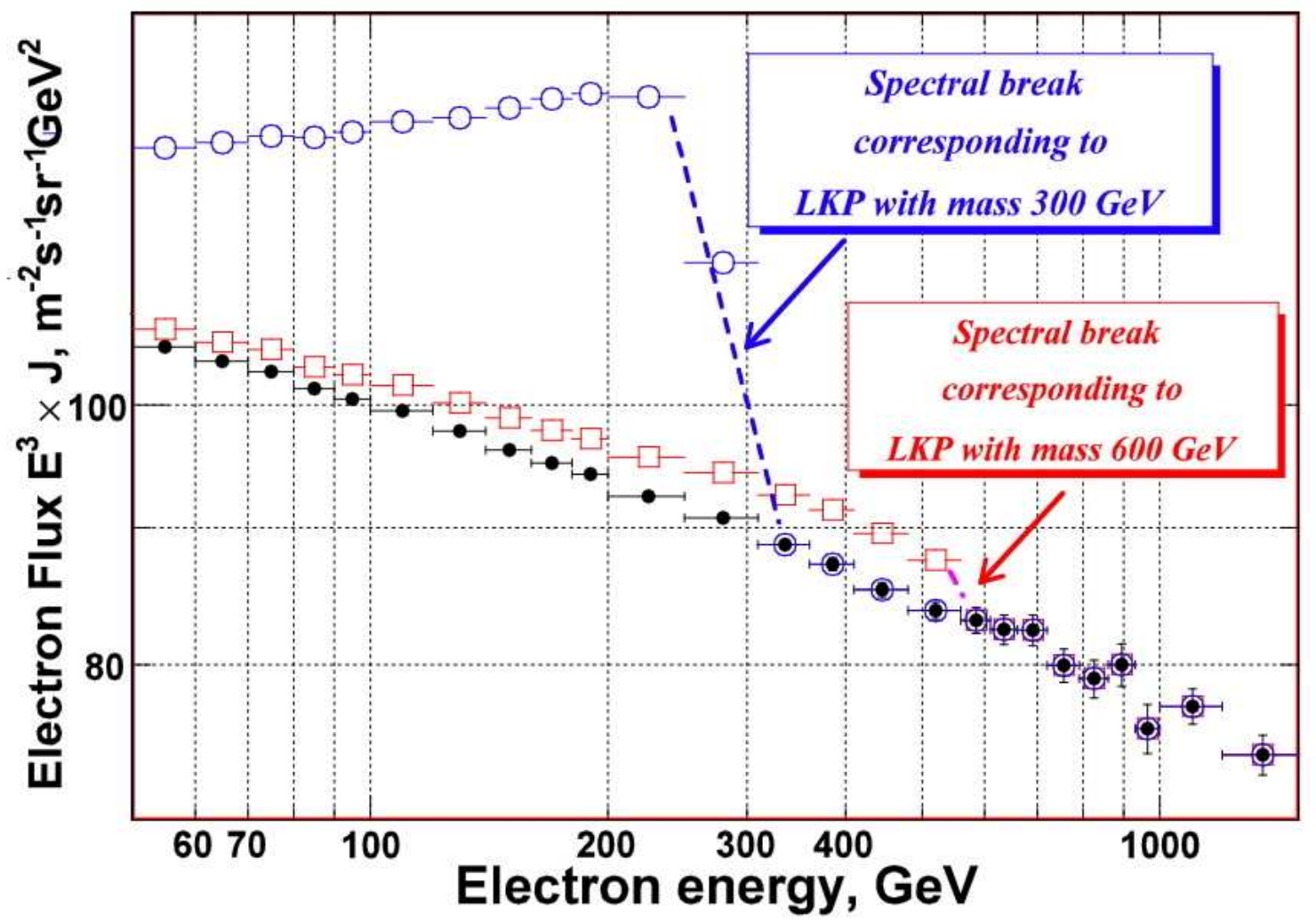

Figure 5: Simulated detection of lightest Kaluza-Klein particles (LKPs) with masses of 300 and $600 \mathrm{GeV}$ in the LAT electron spectrum to be collected in five years of operation. Filled circles: "conventional" electron flux; open circles: the same but with added signal from $300 \mathrm{GeV}$ LKPs; open squares: the same with added signal from $600 \mathrm{GeV}$ LKPs.

\section{References}

[1] P. Picozza et al. [PAMELA Coll.], Astropart. Phys. 27 (2007) 296 [astro-ph/0608697]

P. Picozza and A. Morselli, J. Phys. G: Nucl. Part. Phys. 29 (2003) 903 [astro-ph/0211286]

P. Picozza and A. Morselli, World Scientific Publishing Co. [astro-ph/0604207]

[2] O. Adriani et al. [PAMELA Coll.], arxiv:0810.4994

[3] A. W. Strong, I. V. Moskalenko, and V. S. Ptuskin, Annu. Rev. Nucl. Part. Sci. 57 (2007) 285

[4] A. W. Strong and I. V. Moskalenko, ApJ 509 (1998) 212

I. V. Moskalenko and A. W. Strong, ApJ 493 (1998) 694

[5] I. V. Moskalenko et al., ApJ 565 (2002) 280

[6] A. Lionetto, A. Morselli, and V. Zdravkovic, J. Cosmol. Astropart. Phys. JCAP09 (2005) 010 [astro-ph/0502406] 
[7] V. S. Ptuskin et al., ApJ 642 (2006) 902

[8] K. Abe et al. [BESS Coll.], arXiv:0805.1754

[9] A. Morselli, ICHEP 2006, 33rd Int. Conf. 2007 Word Scientific, pg 222-225, SBN-13 978-981-270-385-9

[10] A. Morselli at al., Nucl. Phys. 113B (2002) 213

[11] A. Cesarini, F.Fucito, A.Lionetto, A.Morselli, P. Ullio, Astropart. Phys. 21 (2004) 267 [astro-ph/0305075]

[12] D. N. Spergel, et al. [WMAP Coll.], ApJS 170 (2007) 377 [astro-ph/0603449]

[13] H. Baer, et al., JCAP 0408 (2004) 005

[14] O. Adriani et al. [PAMELA Coll.], arxiv:0810.4995

[15] J. M. Clem et al., ApJ 464 (1996) 507

[16] F. A. Aharonian, A. M. Atoyan and H. J. Völk, Astron. Astrophys. 294 (1995) L41

[17] S. Coutu et al., Astropart. Phys. 11 (1999) 429

[18] D. Hooper et al., arXiv:0810.1527

[19] W. B. Atwood et al., ApJ (2008) submitted

[20] E. Baltz et al., JCAP07 (2008) 013 [arXiv:0806.2911]

[21] L. Bergström, T. Bringmann and J. Edsjö, arXiv:0808.3725

[22] N. Arkani-Hamed et al., arXiv:0810.0713

[23] K. Yoshida et al., Adv. Space Res. 42 (2008) 1670

[24] J. Chang, et al., Nature 456 (2008) 362

[25] A. A. Moiseev, E. A. Baltz, J. F. Ormes, and L. G. Titarchuk, arXiv:0711.3033

[26] T. Kobayashi, Y. Komori, K. Yoshida, and J. Nishimura, ApJ 601 (2004) 340 